\title{
Análise biopolítica do discurso oficial sobre educação em saúde para pacientes diabéticos no Brasil'
}

\section{A biopolitical analysis of the official discourse about health education for diabetic patients in Brazil}

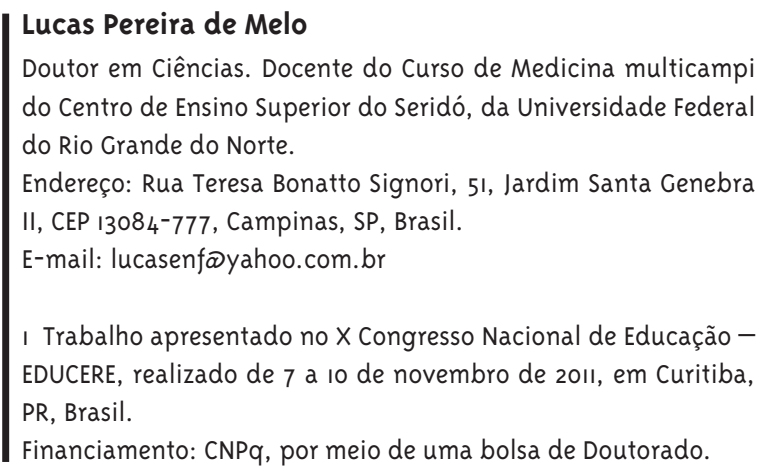

\section{Resumo}

No contexto brasileiro de transição epidemiológica, a educação em saúde tem sido uma ferramenta na luta pela promoção de estilos de vida "saudáveis" e adesão ao tratamento. Dentre essas doenças destaca-se o diabetes mellitus, considerado um problema de saúde pública. O objetivo deste estudo é analisar o discurso oficial sobre educação em saúde para diabetes mellitus, na primeira década dos anos 2000, no Brasil, fundamentado nos conceitos de biopolítica e práticas discursivas de Michel Foucault. Foi feita a revisão bibliográfica de dois documentos publicados pelo Ministério da Saúde brasileiro relacionados ao tema em estudo: "Manual de hipertensão arterial e diabetes mellitus", de 2002; e o "Caderno de atenção básica, número 16, diabetes mellitus”, de 2006. Essas publicações têm orientado as práticas dos profissionais de saúde nos últimos dez anos. A localização e obtenção das fontes foram realizadas no site da Biblioteca Virtual do Ministério da Saúde, em dezembro de 2010. São discutidas as características gerais dos discursos oficiais; os objetivos das ações educativas para pacientes diabéticos; as temáticas abordadas nessas ações; o imperativo técnico e moral que rege o incentivo à realização das práticas educativas; e os parâmetros para avaliação e acompanhamento dos adoecidos. Em seguida, expõe-se a configuração do campo de lutas e resistências no interior da educação em saúde para essa população. A análise do discurso oficial possibilitou a compreensão crítica e a produção de questionamento sobre a complexa rede de relações dos objetos postos em discurso nas práticas educativas em saúde para pacientes diabéticos no Brasil.

Palavras-chave: Educação em saúde; Diabetes mellitus; Biopolítica; Discursos; Prática de saúde pública. 


\section{Abstract}

In the Brazilian context of epidemiologic transition, chronic illnesses have assumed a special place in healthcare policies. In this sense, health education has been a tool to promote "healthy" life-styles and adhesion to treatment. Among those illnesses, diabetes mellitus stands out. Currently, diabetes is regarded as an important public health problem. Given this fact, the aim of this study was to analyze the official discourse about diabetics' health education in the years 2000 in Brazil. The analysis was guided by the concepts of biopolitics and discursive practices of Michel Foucault. A bibliographic review was conducted on two documents published by Brazilian Government: the Manual on Hipertension and Diabetes mellitus (Manual de Hipertensão arterial e Diabetes mellitus), 2002; and the Primary Care notebook no. 16 (Diabetes Mellitus) (Caderno de Atenção Básica, número 16, Diabetes Mellitus), 20o6. These publications have oriented the practices of healthcare professionals over the last 10 years. The documents were obtained at the website of the Virtual Library of the Brazilian Healthcare Ministry. The general features of the official discourse; the aims of health education actions to people with diabetes; the thematic broached in these actions; the technical and moral imperative to carry out the educational practices and the parameters for evaluation and follow-up of the patients were analyzed. Hereafter, the configuration of tensions and resistances in the health education field for the population were exposed. The analysis of the official discourse allows a critical comprehension and raises questions about the complex relationships between the objects which are put on discourse in educational practices on health for people with diabetes in Brazil.

Keywords: Health Education; Diabetes Mellitus; Biopolitics; Discourses; Public Health Practices.

\section{Introdução}

A sociedade atual tem convivido com uma noção de saúde aliquid, ou seja, a saúde "coisa”, um valor, um bem de consumo a ser possuído no "mercado" biotecnológico, do fitness, da medicina estética, dos alimentos diet e light e das griffes. Há um enfoque no sujeito autônomo e individual e no seu estilo de vida, o que tem sido levado ao extremo no contexto brasileiro de transição epidemiológica, no qual as doenças crônicas não transmissíveis têm assumido os primeiros lugares dos boletins epidemiológicos.

O imperativo moral por mudanças e adequações nos estilos de vida da população tem reclamado estratégias e intervenções direcionadas à mudança de comportamentos e adesão a hábitos de vida "saudáveis”. Nesse sentido, a educação em saúde tem sido solicitada pelos gestores das políticas públicas como ferramenta na luta contra os "vilões"do bem-estar.

Com isso, as práticas de educação em saúde ampliam sua permeabilidade e capilaridade no cotidiano de vida das comunidades. Entretanto, os avanços apontados pela história oficial da educação em saúde no Brasil (Levy e col., 1997), contrapõem-se às nossas vivências de profissionais de saúde, no cotidiano de trabalho com grupos e na prática docente em enfermagem (Melo e col., 2011). Nesses cenários, veem-se despontar teimosamente os indícios da má utilização de práticas pedagógicas que, transvertidas em "educação em saúde", mostram-se erroneamente aplicadas no cotidiano de trabalho dos profissionais de saúde (Meyer e col., 2006; Hull, 2008; Melo e col., 2011).

Observa-se que a concepção biomédica e mecanicista do processo saúde-doença, presente desde os primórdios da educação sanitária no Brasil, persiste, ainda que veladamente, nos cursos de formação profissional em saúde. Segundo Besen e colaboradores (2007), esses profissionais reproduzem em suas práticas tais concepções, que, no caso da educação em saúde, mostram-se patologizantes, verticalizadoras e culpabilizadoras. Ou seja, os profissionais, alienados pela prática biomédica, não encontram caminhos capilares para a transformação de sua prática social.

Nossa experiência com grupos para adoecidos por diabetes mellitus (DM) na atenção básica e 
a literatura especializada têm demonstrado os investimentos dos pesquisadores e profissionais na inovação de suas práticas educativas. Porém, a maior parte desses esforços tem-se concentrado na criação de novas tecnologias educativas (Torres e col., 2003; Otero e col., 2008; Vincent, 2009; Oliveira e col., 2009). Portanto, paralelo ao incremento "pedagógico", há a necessidade de um aprofundamento epistemológico dos fundamentos de tais práticas, já que se considera que toda prática educativa se constrói com intencionalidade.

Essas vivências nos têm feito acreditar na existência de um "pano de fundo" que historicamente tem sustentado essas práticas, ao mesmo tempo em que, em dados contextos, impede ou dificulta a produção de cuidados congruentes com as necessidades de saúde do usuário. No nosso entender, esses "panos de fundo" podem ser considerados como práticas discursivas, no sentido foucaultiano (Foucault, 2010a).

Para esse autor, as práticas discursivas são os lugares onde se formam ou se deformam, onde aparecem e se apagam séries emaranhadas de objetos - como jogos de diferenças, de desvios, de substituições, de transformações, ao mesmo tempo superpostas e lacunares. Esses objetos não são produzidos no interior desses discursos, mas em determinadas condições de externalidade que lhes permitem aparecer, justapor-se a outros objetos, situar-se em relação a eles, definir sua diferença, sua irredutibilidade e, eventualmente, sua heterogeneidade; enfim, serem colocados em um campo de externalidade (Foucault, 2010a).

Portanto, as práticas discursivas não se produzem e se encerram em si mesmas. Igualmente não há o papel positivo do autor ${ }^{2}$, da disciplina, da vontade de saber. 0 que há, é um recorte e uma rarefação ${ }^{3}$ do discurso (Foucault, 2010b). Essas práticas inserem-se, em todo caso, em um contexto de relações que reclama as instâncias de emergência, de delimitação e de especificação desses discursos.
Nessa perspectiva, os discursos oficiais sobre educação em saúde não têm o objetivo único e altruísta da promoção da saúde, da "salvação" ou do prolongamento da vida. Trata-se de um discurso que recai sobre a vida e suas formas de ser conduzida, produzindo tecnologias reguladoras, normas e padrões para a população e sua multiplicidade de processos vitais. Nesse sentido, segundo Gastaldo (1997), a educação em saúde se mostra como uma experiência de ser governado por outros e uma solicitação de autogoverno e autodisciplina, ou seja, é uma estratégia biopolítica.

O termo "biopolítica" designa o que faz a vida e seus mecanismos entrar no domínio dos cálculos explícitos, e faz do poder-saber (biopoder) um agente de transformação da vida humana. Trata-se da assunção da vida pelo poder - uma tomada de poder sobre o homem enquanto ser vivo, uma espécie de estatização do biológico (Foucault, 2002, 2010c).

Portanto, parte-se do pressuposto de que há relações entre as práticas discursivas produzidas oficialmente no campo da educação em saúde e o insucesso das ações educativas realizadas pelos profissionais na atenção básica. Pois as práticas discursivas que operam nas ações de educação em saúde, tal como se tem colocado historicamente, dificultam a produção singular de cuidados, prejudicando a emergência de estilos de vida distintos daqueles preconizados pelo modelo biomédico cartesiano e a política de saúde nele inspirada.

Diante da complexidade de áreas e temas na educação em saúde foi realizado um recorte empírico. Logo, as práticas discursivas analisadas serão aquelas voltadas aos portadores de DM e que se desenvolvem nos equipamentos da rede básica de serviços de saúde. Isso devido à importância da enfermidade no conjunto das doenças crônicas, caracterizando-se como um problema de saúde pública (Meetoo, 2008) e pela nossa experiência de ensino, pesquisa e assistência na enfermagem em saúde coletiva.

\footnotetext{
2 Entende-se por autor não o indivíduo falante que pronunciou ou escreveu um texto (como na abordagem tradicional), mas o autor como princípio de agrupamento do discurso, como unidade e origem de suas significações, como foco de sua coerência (Foucault, 201ob).

3 "Rarefação dos sujeitos que falam; ninguém entrará na ordem do discurso se não satisfizer a certas exigências ou se não for, de início, qualificado para fazê-lo. Mais precisamente: nem todas as regiões do discurso são igualmente abertas e penetráveis; algumas são altamente proibidas (diferenciadas e diferenciantes), enquanto outras parecem quase abertas a todos os ventos e postas, sem restrição prévia, à disposição de cada sujeito que fala" (Foucault, 2010b, p. 37).
} 
O objetivo deste estudo é analisar o discurso oficial sobre educação em saúde para diabetes mellitus, no Brasil, desde uma perspectiva biopolítica fundamentada na obra de Michel Foucault.

\section{Discurso oficial sobre educação em saúde para diabetes no Brasil: uma análise biopolítica}

Uma revisão dos documentos sobre educação em saúde emitidos pelo Ministério da Saúde brasileiro, de 1980 a 1992, revelou uma transformação do discurso oficial: o deslocamento de uma perspectiva educativa tradicional e normativa para outra, participativa, a partir de uma abordagem construtivista. Os documentos analisados diziam respeito às diretrizes gerais para a educação em saúde, no período estudado, sem especificar uma temática ou grupo populacional específico (Gastaldo, 1997).

A análise do discurso oficial sobre educação em saúde para DM no Brasil, proposta neste trabalho, foi realizada com base em duas publicações ministeriais: "Manual de hipertensão arterial e diabetes mellitus" (Brasil, 2002), integrante do Plano de Reorganização da Atenção à Hipertensão Arterial e ao Diabetes Mellitus; e "Diabetes mellitus" (Brasil, 2006), referente ao número 16 da Coleção Cadernos de Atenção Básica. A obtenção dessas fontes se deu por meio de buscas no site da Biblioteca Virtual do Ministério da Saúde, em dezembro de 2010.

Essas publicações têm norteado as práticas dos profissionais de saúde na atenção básica, nos últimos dez anos. Foram a partir delas que Estados e municípios criaram seus protocolos de atendimento para portadores de hipertensão arterial e diabetes mellitus. 0 recorte cronológico fundamentou-se no período em que foi criado o Plano de Reorientação da Atenção à Hipertensão Arterial (HA) e ao Dibetes Mellitus, em 200o, e no Sistema de Cadastramento e Acompanhamento de Hipertensos e Diabéticos (SISHIPERDIA), em 2002. Esses dois eventos marcam, de forma decisiva, a organização e funcionamento da educação em saúde para DM na atenção básica, desde então.

Após leitura cuidadosa e interessada desses materiais, com o objetivo de identificar os trechos nos quais havia referências à educação em saúde, foi produzido um corpus para análise e categorização das informações obtidas (Gil, 1999).

Cumpre ressaltar que não foi nosso objetivo fazer oposição às diretrizes propostas pelo Ministério da Saúde; pelo contrário, o que se propõe é estranhar, desnaturalizar e problematizar o presente, aquilo que nos é dado como natural, ou seja, "o jeito como as coisas sempre funcionaram por aqui", a maneira como aprendi a "ensinar" e como aprendi a fazer e saber educação em saúde.

A seguir são apresentadas as categorias temáticas que emergiram da análise, acompanhadas de uma discussão com a literatura.

\section{"Em pouco, muito se diz": uma visão geral dos documentos}

O conteúdo das duas publicações conserva características em comum em termos de conteúdo, de estilo de linguagem, de recomendações e de enfoques. De forma geral, os conteúdos dos manuais trazem indicações prescritivas, focadas na redução de fatores de risco, e forte argumentação, fundamentada em resultados de pesquisas nacionais e internacionais. Há um estilo de linguagem direta, objetiva e imperativa com grande poder de convencimento.

O ponto alto dos textos é o direcionamento das ações para o indivíduo - sujeito individual, autônomo, responsável, racional, pensante e consciente (Hall, 2011). Tal posicionamente reflete, até certo ponto, a influência do movimento de promoção da saúde, em suas fases iniciais, cujo centro das discussões em muito se aproximava da produção de autonomia com vistas ao autocuidado. Muitas dessas discussões têm sido repensadas por teóricos do campo da saúde coletiva, uma vez que os desdobramentos dessa perspectiva produziram um tipo de culpabilização velada nas práticas de saúde em geral e, especialmente, nas práticas educativas.

Dessa forma, tanto os indivíduos adoecidos por DM quanto a equipe multiprofissional de saúde são responsabilizados pela promoção e melhoria do estado de saúde. Todas as normatizações e diretrizes dadas pelos documentos são ancoradas em dados de pesquisas clínicas e de impactos socioeconômicos. Diante das justificativas oferecidas, a mensagem do texto "impregna-se" no leitor de forma subliminar; e a consequência disso, geralmente, é a reprodução mecanizada das informações, ofuscando o pensa- 
mento crítico do profissional.

O enfoque pautado no modelo biomédico e na "epidemiologia do risco" ganha um adicional poderoso no contexto atual, marcado pela disseminação em massa de informações de todos os tipos: a evidência científica!

Nesses discursos, as variadas dimensões e os aspectos experienciais da enfermidade, de natureza individual e coletiva, são secundarizados ou omitidos. Em nossa interpretação, diante desse conjunto de características observadas nos materiais, configura-se uma possibilidade de compreensão das posturas tecnicistas assumidas pelos profissionais de saúde, já que estes se colocam, na maioria das vezes, como reprodutores de tais discursos.

O tema que perpassa esses documentos, no tocante à educação em saúde, é a aderência ao tratamento proposto e a estilos de vida "saudáveis". Nessa conjuntura, o que é "saudável" é aquilo que a política de saúde diz ser saudável. Em outras palavras, a educação em saúde, da forma como é definida pelas diretrizes do Ministério da Saúde, objetiva reforçar padrões de saúde concebidos pelo governo para a população. Tais padrões podem ser entendidos como uma estratégia de governabilidade através da biopolítica (Gastaldo, 1997).

Dessa forma, os documentos retomam dois enfoques centrais do biopoder: a noção de população (problema a um só tempo político, científico, biológico e de poder) e o estabelecimento de "mecanismos reguladores" que, nessa população global com seu campo aleatório, vão poder fixar um equilíbrio, manter uma média, estabelecer uma espécie de homeostase, assegurar compensações (Foucault, 2002).

\section{0 objetivo das ações educativas para pacientes diabéticos}

A educação é considerada essencial ao tratamento e tida como um direito e dever do indivíduo adoecido e um dever do profissional de saúde (Brasil, 2002). Cabe, então, ao paciente e aos seus familiares a participação e interesse nas questões relacionadas ao seu processo de adoecimento e tratamento. Já para os profissionais de saúde a tarefa fica centrada no imperativo técnico e moral de realizar ações educativas que enfatizem o empoderamento do paciente e o desenvolvimento de autonomia e ca- pacidades para o autocuidado (Brasil, 2002, 2006). Em síntese, o objetivo da educação em saúde para DM é promover a efetividade e adesão do paciente às ações e intervenções propostas nos protocolos, sempre considerando a produção de autonomia do adoecido (Brasil, 2002, 2006).

Pode-se observar que, embora os protocolos e padrões que normatizam o tratamento desses indivíduos sejam direcionados a um fenômeno populacional, coletivo - uma biopolítica da espécie humana -, seu objetivo último é atingir, capilarmente, os corpos individuais. Logo, essa estratégia biopolítica, quando levada a seu extremo, é capilar, corporal, detalhista e minuciosa, incorporando as práticas da anatomopolítica do corpo humano que caracteriza o poder disciplinar (Foucault, 2002).

\section{0 que se ensina sobre diabetes no Brasil}

Partindo-se do pressuposto de que vivemos sob um "estado de alerta" acionado pelo Estado, diante dos riscos das doenças cardiovasculares, que têm o DM como um de seus principais fatores de risco, os discursos oficiais indicam a necessidade de "mantermos os olhos fixos" no conjunto de elementos relacionados a essas enfermidades. Nessa perspectiva, claramente biológica e patologizante, há um esvaziamento das outras dimensões que participam dialeticamente do processo saúde-doença.

Configura-se, assim, uma tríade temática: comer, exercitar, medicar! No "Manual de hipertensão arterial e diabetes mellitus" (Brasil, 2002) indicam-se os pontos que devem ser abordados nas ações de educação em saúde:1) informar sobre as consequências do DM e HA não tratados ou mal controlados; 2) reforçar a importância da alimentação como parte do tratamento; 3) esclarecer sobre crendices, mitos, tabus e alternativas populares de tratamento; 4) desfazer temores, inseguranças e ansiedade do paciente; 5) enfatizar os benefícios da atividade física; 6) orientar sobre hábitos saudáveis de vida; 7) ressaltar os benefícios da automonitoração, insistindo no ensino de técnicas adequadas e possíveis; 8) ensinar como o paciente e sua família podem prevenir, detectar e tratar as complicações agudas em casa, até chegar ao hospital ou ao posto de saúde mais próximo; 9) ensinar claramente como detectar os sintomas e sinais de complicações crônicas, em particular nos 
pés; 10) ressaltar a importância dos fatores de risco cardiovasculares; 11) incentivar o paciente a se tornar mais autossuficiente no seu controle.

Dessa forma, sem negar a importância desses pontos, pode-se considerar que aí opera uma lógica terapêutica prescritiva e normativa. Sendo assim, enfatiza a gestão da vida por meio de uma abordagem coletiva (porém, ao mesmo tempo, individualista) que objetiva a promoção do cuidado de si.

Já no Caderno de Atenção Básica (Brasil, 2006) aparecem indicações de que essas atividades educativas sejam de cunho multidisciplinar e considerem os aspectos culturais e psicossociais. No entanto, não são discutidos no documento esses últimos aspectos.

Em todos esses pontos temáticos permeia a ideia latente de mudanças no comportamento e estilo de vida. Esses temas têm sido recorrentes na história da educação em saúde no Brasil, embora historicamente tenham cambiado e/ou justaposto os objetos de suas normatizações (Melo, 1976; Candeias, 1988; Gastaldo, 1997).

O “comportamento saudável” é apresentado como a norma, e todos os outros comportamentos tornam-se desviantes, sendo, por isso, geradores de culpa no "transgressor". O princípio que está por trás da norma é o de que alguém, além do indivíduo, conhece melhor o que é apropriado ou "bom para todos". Esse "alguém" tende a ser incorporado pelo profissional de saúde, que passa a se enxergar como uma autoridade sanitária, porta-voz desse discurso "são". Esse mesmo princípio, também experienciado em outras áreas da vida social, é o que produz corpos governáveis (Gastaldo, 1997).

\section{Ações de educação em saúde: um imperativo técnico e moral}

Os documentos são contundentes em suas explanações que caracterizam o DM como uma epidemia em curso. As "evidências científicas" não deixam dúvidas do dever que todo profissional de saúde tem diante da ameaça ao estado de saúde do brasileiro. Imbuídos desse imperativo técnico (pelas suas inserções profissionais) e moral (pela sua condição de cidadão brasileiro diante de um "risco ameaçador” à saúde da nação) os trabalhadores em saúde são estimulados a realizar: campanhas educativas periodicamente, grupos de pacientes hipertensos e diabéticos, grupos de ajuda mútua (caminhada, trocas de receitas, autocuidado etc.) e ações comunitárias e governamentais para promover estilos de vida saudáveis (Brasil, 2002, 2006).

0 médico deve, além das ações anteriores, "perseguir, obstinadamente, os objetivos e metas do tratamento (níveis pressóricos, glicemia pós-prandial, hemoglobina glicada, controle de lipídeos e do peso)" (Brasil, 2002, p. 87). Talvez a análise crítica dessa afirmativa nos sirva para compreender as práticas dos profissionais de saúde que enfatizam esses parâmetros no atendimento aos usuários e as consequentes frustrações diante do insucesso da terapêutica e o reforço da sensação de fracasso do doente diante de sua "incapacidade" ou "descomprometimento" com seu autocuidado.

Há, também, nesses discursos recomendações para que essas ações utilizem metodologias participativas. Esse tipo de recomendação já vem sendo pontuada desde os anos 1980 (Gastaldo, 1997) e, atualmente, tem ganhado força com o Movimento da Educação Popular em Saúde. Em termos da biopolítica, isso significa que o discurso sobre educação em saúde ultrapassa as estratégias repressivas - tais como palestras, exigências em termos de higiene e hábitos saudáveis compulsórios - para chegar à consolidação de táticas construtivas, tais como as atividades de grupo e construção de conhecimento comum entre profissionais e pacientes (Gastaldo, 1997).

No entanto, o que temos visto no cotidiano dos centros de saúde não condiz com essas mudanças no discurso oficial. Os espaços educativos continuam preenchidos pela voz do trabalhador em saúde (Melo e col., 2011). Nessa conjuntura, os usuários são estimulados a fornecer informações sobre si mesmos. Segundo Foucault (2010c), trata-se da formidável injunção de que devemos dizer o que somos, o que fazemos, o que recordamos e o que foi esquecido, o que e o que se oculta, o que não pensamos e o que pensamos inadvertidamente.

Pelo enfoque biopolítico, a negação de dizer-se (em grupos, nas consultas, nas visitas domiciliárias), de arquivar-se (os "arquivos" são os textos em co-autoria produzidos por pacientes e profissionais de saúde a partir daquilo que se confessa, por 
exemplo, numa consulta e que são registrados em prontuários e outros documentos) caracteriza uma atitude de resistência ao "grande olho" ou efeito panóptico (Foucault, 1984). Essa produção exaustiva de arquivos caracteriza o que Foucault (2010c) chamou de "técnicas de confissão".

Diante disso, concordamos com Gastaldo (1997), ao discutir a participação comunitária nas ações educativas em saúde, pois se trata, veladamente, de uma solicitação de confissão e de obediência às normas e padrões da política de saúde. É, assim, um convite compulsório para se colocar em discurso e, a partir disso, servir como um exemplo a ser seguido ou classificado como um "caso difícil de ser tratado".

\section{Parâmetros de avaliação das ações educativas}

A biopolítica visa dirigir a multiplicidade dos homens, na medida em que ela forma uma massa global, afetada por processos de conjunto que são próprios da vida: nascimento, morte, produção, doença etc. Portanto, tratam-se de doenças mais ou menos difíceis de extirpar (as doenças crônicas, por exemplo), justamente por terem caráter permanente, o que acarreta subtração das forças, diminuição do tempo de trabalho, baixa de energias, custos econômicos, tanto por causa da produção não realizada quanto dos custos dos tratamentos que podem ser gerados (Foucault, 2002).

Nesse contexto, as instruções para avaliação e acompanhamento dos usuários que participam de ações educativas sobre DM dadas pelo discurso oficial pautam-se em parâmetros estatísticos de normalidade comuns na biomedicina positivista. Enfatizam-se, hierarquicamente, critérios bioquímicos, fisiológicos e psicossociais para avaliação da efetividade da ação educativa e a adesão do paciente: controle da glicemia, controle metabólico, ausência de complicações agudas e crônicas, mudanças de hábitos, adaptação psicossocial, aderência ao tratamento (Brasil, 2002).

Dessa forma, chama a atenção o caráter classificatório da biomedicina cartesiana e sua instrumentalização pelas estatísticas vitais ou bioestatística. A biopolítica se utiliza desse instrumental para compreender os fenômenos populacionais e, por meio disso, atingir os corpos individuais (Foucault, 2002). O estabelecimento de tais critérios tem sido relatado na literatura como produtor de frustrações nos profissionais e culpabilização dos usuários diante das dificuldades de atingirem os padrões estabelecidos pela política de saúde (Hull, 2008; Castellanos e col., 2011; Melo e col., 2011).

A noção de controle é predominante nesses processos avaliativos. No entanto, estudos socioantropológicos sobre a experiência e as representações sociais dos adoecidos por DM afirmam que a noção de "controle" associa-se ao gerenciamento da doença. O "controle" apresenta duas concepções: uma biomédica, que significa manter a glicemia e outros parâmetros dentro dos valores de referência; e uma popular, que se volta para as preocupações práticas mobilizadoras dos adoecidos a promoverem ajustes nas prescrições, tentando equilibrá-las em meio às demandas não médicas a serem gerenciadas na vida (Barsaglini, 2007, 2008; Barsaglini e Canesqui, 2010).

Esses dados corroboram a necessidade de serem repensados os critérios avaliativos indicados nos documentos oficiais ou, minimamente, de haver "frestas" nesse emaranhado discursivo que possibilitem ao indivíduo não apenas o direito de "confissão", mas o de ser compreendido e respeitado em seus saberes, fazeres e vivências cotidianas (individuais e coletivas) com a enfermidade crônica.

\section{Eu ensino, você aprende? A configuração de um campo de lutas e resistências}

Diante da configuração dessas tramas discursivas e das estratégias biopolíticas (biopoder) de gerenciamento da vida discutidas até aqui, é de fundamental importância levantar as questões que dizem respeito aos campos de lutas e de resistências existentes no interior das práticas educativas em saúde. Isso porque, na perspectiva foucaultiana, as relações de poder só existem em ato e no campo de possibilidades próprias dos jogos de relações entre sujeitos ou grupo ativos, "livres". As relações de poder não se completam de uma vez por todas; dão-se sempre num imbricamento de lutas imediatas. Da relação entre poder e liberdade emerge (dentro deste emaranhado de relações; e não fora dele) a resistência e sua visceral relação com o poder (Foucault, 1995, 2010c). Portanto: 
Não há relação de poder sem resistência, sem escapatória ou fuga, sem inversão eventual; toda relação de poder implica, então, pelos menos de modo virtual, uma estratégia de luta, sem que para tanto venham a se superpor, a perder sua especificidade e finalmente a se confundir (Foucault, 1995, p. 248).

Os pontos de resistência estão presentes em toda a rede de poder. Portanto, não existe, com respeito ao poder, um lugar da grande Recusa - alma da revolta, foco de todas as rebeliões, lei pura do revolucionário. Mas sim resistências, no plural, que são casos únicos: possíveis, necessárias, improváveis, espontâneas, selvagens, solitárias, planejadas, arrastadas, violentas, irreconciliáveis, prontas ao compromisso, interessadas ou fadadas ao sacrifício; por definição, não podem existir a não ser no campo estratégico das relações de poder (Foucault, 2010c).

Os estudos têm mostrado diversos indícios ou evidências desses campos de lutas e de resistências. Da parte dos usuários pode-se observar: a não adesão aos tratamentos e práticas de cuidados propostas; o silêncio e a indiferença, evidenciados principalmente pela não participação dos usuários nas ações realizadas e pela não incorporação das informações em seus repertórios de preocupações cotidianas; e a ausência ou baixa frequência nos grupos educativos, nas palestras, nos eventos com propostas educativas (Péres e col., 2007; Barsaglini, 2007; Hull, 2008; Otero e col., 2008; Melo e col., 2011).

No que se refere aos profissionais de saúde pode-se enumerar: o despreparo pedagógico; o não planejamento das ações educativas; a reprodução de posturas autoritárias nas suas práticas educativas; os sentimentos de frustração e impotência diante da realidade resistente às mudanças pro(im)postas; e o desenvolvimento de práticas educativas que produzem um sentimento de culpabilização no usuário pelo insucesso do seu tratamento e do seu jeito de andar a vida (Torres e col., 2003; Teixeira e Zanetti, 2006; Hull, 2008; Oliveira e col., 2009).

Essas resistências e lutas são engendradas nos tensos espaços de produção de cuidados em saúde.
Espaços de relações ${ }^{4}$ nos quais interagem atores sociais diversos. Resistências e lutas que, sem passar pelos crivos das classificações polarizantes (positivas/negativas, boas/más, certas/erradas, produtivas/improdutivas), podem ser consideradas como a força motriz que possibilita a emergência das potências dos encontros.

\section{Considerações finais}

A análise dos discursos oficiais sobre educação em saúde para DM, no Brasil, por meio dos conceitos foucaultiano de práticas discursivas e biopolítica, possibilitou a compreensão crítica da complexa rede de relações entre os objetos colocados em discurso nas práticas educativas em saúde.

Nesse movimento, examinar esses discursos oficiais, por meio desse referencial teórico, é uma maneira de analisar os diferentes modos pelos quais os indivíduos adoecidos, alvos dessas ações educativas, tornam-se sujeitos. Trata-se mesmo de problematizar o presente e nossos modos de operar enquanto profissionais de saúde encarregados da difícil e, quem sabe, utópica missão de "promover" a saúde da população.

O discurso oficial pesquisado traz as marcas do modelo biomédico e da "epidemiologia do risco": enfoques biologicista, patologizante, prescritivo, normatizador, generalizador (embora individualizante) e fragmentador dos indivíduos e de suas realidades socioculturais. Efeito de seus objetivos, a obstinação pela adesão ao tratamento mecaniciza a prática dos profissionais e "persegue" os usuários em um jogo de lutas e resistências (onde há poder, há resistência). Evidenciaram-se também as ações propostas e os meios de avaliação empregados, e a abordagem, por meio de práticas verticalizadoras e comportamentais, do "sobe-e-desce" dos parâmetros clínicos utilizados. Nesse conjunto destaca-se a tríade temática "comer, exercitar, medicar!" que baliza os eixos fundamentais das práticas educativas em diabetes.

4 Cumpre lembrar a relevante produção acadêmica de Emerson Elias Merhy a respeito desses espaços de relações. Merhy (2004) discute a existência de uma micropolítica de encontros, sendo estes de dois tipos: encontros de interdição/dominação e os encontros autopoiéticos (representa o movimento da vida que produz vida). No mesmo estudo o autor discute a noção de relações intercessoras como aquelas produtoras de cuidado em saúde. 
À guisa de conclusão, espera-se que a crítica aos discursos oficiais trazidos à tona neste estudo tenha a potência de nos fazer problematizar o presente: "o que nos acontece agora, quem somos agora, o que podemos pensar e o que podemos dizer e o que podemos experimentar agora, neste exato momento da história" (Larrosa, 2004, p. 34). Ou, simplesmente, nos questionar: quais os efeitos produzidos pela educação em saúde, do jeito como está dada? Há outras formas de produzir tais efeitos? Por que realizamos ações de educação em saúde? Por que fazemos dessa forma e não de outra?

\section{Referências}

BARSAGLINI, R. A. "Com açúcar no sangue até o fim": um estudo de caso sobre o viver com diabetes. In: CANESQUI, A. M. (Org.). Olhares socioantropológicos sobre os adoecidos crônicos. São Paulo: Hucitec: Fapesp, 2007. p. 53-85.

BARSAGLINI, R. A. Análise socioantropológica da vivência do diabetes: um estudo de caso. Interface: Comunicação, Saúde, Educação, Botucatu, v. 12, n. 26, p. 563-577, 2008.

BARSAGLINI, R. A.; CANESQUI, A. M. A alimentação e a dieta alimentar no gerenciamento da condição crônica do diabetes. Saúde e Sociedade, São Paulo, v. 19, n. 4, p. 919-932, 2010.

BESEN, C. B. et al. A Estratégia Saúde da Família como objeto da educação em saúde. Saúde e Sociedade, São Paulo, v. 16, n. 1, p. 57-68, 2007.

BRASIL. Ministério da Saúde. Secretaria de Políticas de Saúde. Departamento de Ações Programáticas Estratégicas. Plano de reorganização da atenção à hipertensão arterial $e$ ao diabetes mellitus: manual de hipertensão arterial e diabetes mellitus. Brasília, DF, 2002.

BRASIL. Ministério da Saúde. Secretaria de Atenção à Saúde. Departamento de Atenção Básica. Diabetes mellitus. Brasília, DF, 2006. (Cadernos de Atenção Básica, n. 16 - Série A: Normas e Manuais Técnicos).
CANDEIAS, N. M. F. Evolução histórica da educação em saúde como disciplina de ensino na Faculdade de Saúde Pública da Universidade de São Paulo: 1925 a 1967. Revista de Saúde Pública, São Paulo, v. 22, n. 4, p. 347-365, 1988.

CASTELLANOS, M. E. P. et al. Evidências produzidas por pesquisas qualitativas sobre diabetes tipo 2: revisão da literatura. Interface: Comunicação, Saúde, Educação, Botucatu, v. 15, n. 36, p. 257-273, 2011.

FOUCAULT, M. Vigiar e punir. Petrópolis: Vozes, 1984.

FOUCAULT, M. O sujeito e o poder. In: DREYFUS, H. L.; RABINOW, P. (Org.). Michel Foucault, uma trajetória filosófica: para além do estruturalismo e da hermenêutica. Rio de Janeiro: Forense Universitária, 1995. p. 231-249.

FOUCAULT, M. Em defesa da sociedade: curso no Collège de France, ano 1975-1976. São Paulo: Martins Fontes, 2002.

FOUCAULT, M. A arqueologia do saber. 7. ed. Rio de Janeiro: Forense Universitária, $2010 a$.

FOUCAULT, M. A ordem do discurso: aula inaugural no Collège de France, pronunciada em 2 de novembro de 1970. 20. ed. São Paulo: Edições Loyola, 2010b.

FOUCAULT, M. História da sexualidade I: a vontade de saber. Rio de Janeiro: Graal, 2010c.

GASTALDO, D. É a educação em saúde “saudável”?: repensando a educação em saúde através do conceito de bio-poder. Educação \& Realidade, Porto Alegre, v. 22, n. 1, p. 147-168, 1997.

GIL, A. C. Métodos e técnicas de pesquisa social. 5 . ed. São Paulo: Atlas, 1999.

HALL, S. A identidade cultural na pósmodernidade. 11. ed. Rio de Janeiro: DP\&A, 2011.

HULL, L. Diabetes mellitus: a case history of community care. Journal of Community Nursing, Surrey, v. 22, n. 4, p. 4-11, 2008.

LARROSA, J. A operação ensaio: sobre ensaiar e o ensaiar-se no pensamento, na escrita e na vida. Educação \& Realidade, Porto Alegre, v. 29, n. 1, p. 27-43, 2004. 
LEVY, S. N. et al. Educação em saúde: histórico, conceitos e propostas. Brasília, DF, 1997.

MEETOO, D. Chronic diseases: the silent global epidemic. British Journal of Nursing, London, v. 17, n. 21, p. 1320-1325, 2008.

MELO, J. A. C. A prática de saúde e a educação. 1976. Tese (Doutorado em Saúde Pública) Faculdade de Ciências Médicas da Universidade Estadual de Campinas, Campinas, 1976.

MELO, L. P. et al. A experiência de estudantes de enfermagem em um grupo de educação em saúde: uma abordagem dialógica. Revista Brasileira em Promoção da Saúde, Fortaleza, v. 24, n. 2, p. 18o188, 2011.

MERHY, E. E. Cuidado com o cuidado em saúde: saiba explorar seus paradoxos para defender a vida: o ato de cuidar é um ato paradoxal: pode aprisionar ou liberar. Campinas: [s.n.], 2004. Disponível em: <http://www.yumpu.com/pt/ document/view/12817362/cuidado-com-o-cuidadoem-saude-saiba-explorar-seus-uff $>$. Acesso em: 12 set. 2013 .

MEYER, D. E. E. et al. "Você aprende: a gente ensina?": interrogando relações entre educação e saúde desde a perspectiva da vulnerabilidade. Cadernos de Saúde Pública, Rio de Janeiro, v. 22, n. 6, p. 1335-1342, 2006.
OLIVEIRA, N. F. de et al. Fatores terapêuticos em grupos de diabéticos. Revista da Escola de Enfermagem da USP, São Paulo, v. 43, n. 3, p. 558$565,2009$.

OTERO, L. M.; ZANETTI, M. L.; OGRIZIO, M. D. Conhecimento do paciente diabético acerca de sua doença, antes e depois da implementação de um programa de educação em diabetes. Revista Latino-Americana de Enfermagem, Ribeirão Preto, v. 16, n. 2, p. 231-237, 2008.

PÉRES, D. S. et al. Dificuldades dos pacientes diabéticos para o controle da doença: sentimentos e comportamentos. Revista Latino-Americana de Enfermagem, Ribeirão Preto, v. 15, n. 6, p. 11051112, 2007.

TEIXEIRA, C. R. S.; ZANETTI, M. L. O trabalho multiprofissional com grupo de diabéticos. Revista Brasileira de Enfermagem, Brasília, DF, v. 59, n. 6, p. 812-817, 2006.

TORRES, H. C.; HORTALE, V. A.; SCHALL, V. A experiência de jogos em grupos operativos na educação em saúde para diabéticos. Cadernos de Saúde Pública, Rio de Janeiro, v. 19, n. 4, p. 10391047, 2003.

VINCENT, D. Culturally tailored education to promote lifestyle change in Mexican Americans with type 2 diabetes. Journal of the American Academy of Nurse Practitioners, Austin, v. 21, n. 9, p. 520-527, 2009.

Recebido em: 15/01/2012

Reapresentado em: 08/04/2013

Aprovado em: 13/05/2013 